\title{
Application of magnetic technology in local quail house and hatchery on performance, reproductive and physiological traits under heat stress
}

\author{
M.A. Mustafa \\ Animal Resources Department, College of Agriculture Engineering Sciences, Salahaddin University, Erbil, Iraq \\ mahbuba.mustafa@su.edu.krd
}

(Received July 30, 2018; Accepted September 14, 2018)

\begin{abstract}
Using magnetic technology has shown major potentials in different fields especially agriculture. Two experiments were conducted to investigate the using of magnetic technology application in local quail house and hatchery on productive and physiological traits under heat stress condition. Results revealed that quail when served $0.2 \mathrm{~T}$ magnetically treated water (MTW) were significantly $(\mathrm{P} \leq 0.05)$ better in most productive, reproductive characteristics in the $1^{\text {st }}$ experiment: eggs production, water consumption, egg weight, shell strength, estrogen conc., also improved FCR in females, all semen quality and testosterone conc. in males. Also, total RBCs count, thrombocyte, blood pH, blood viscosity, plasma viscosity, blood iron $(\mathrm{Fe})$, copper $(\mathrm{Cu})$, calcium $(\mathrm{Ca})$, thyroxin hormone and total count of Lactobacillus spp. in small intestine. However, reduction in dead sperm, abnormal acrosome, corticosterone conc., body temperature, blood clotting time, E. coli and fungi counts in both sexes. In the $2^{\text {nd }}$ experiment using of magnetic technology on embryonic development traits of local quails incubating eggs that collected from experiment 1 were significantly $(\mathrm{P} \leq 0.05)$ higher in the most characteristics at the group of $0.2 \mathrm{~T}$ MTW as fertility, hatchability, chicks weight, immune status of hatched chicks. While reduction in dead embryo and heterophil-to- lymphocyte (H/L) ratio. Finally, the results of 0.2 T MTW achieved superiorly gains compared with the other groups.
\end{abstract}

Keyword: Magnetic technology, Quail, Hatchery, Reproductive, Physiological traits.

Available online at http://www.vetmedmosul.com

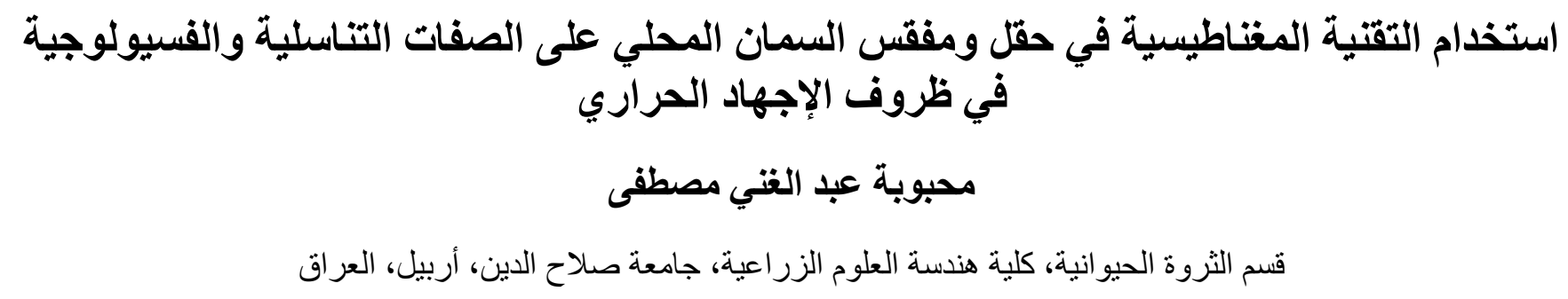

الخلاصة

أن استخدام التقنية المغناطيسية أظهرت كفاءة عالية في مختلف المجالات وخاصة الزراعة الهية أجريت تجربتان للتحقق من استخدام

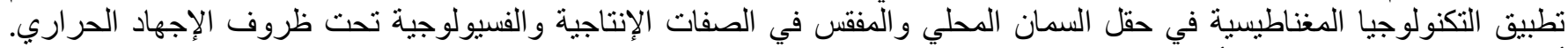

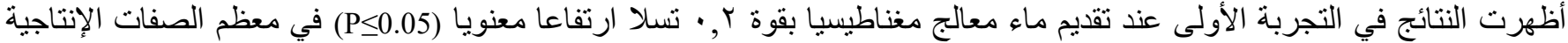

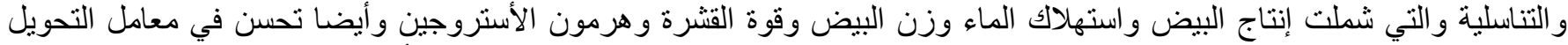

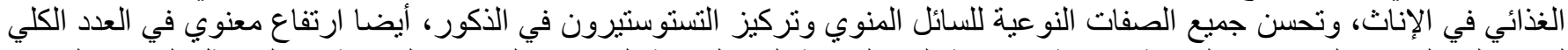

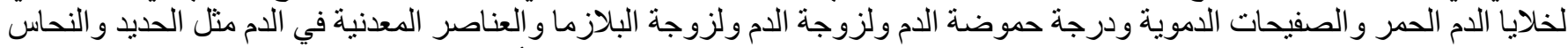

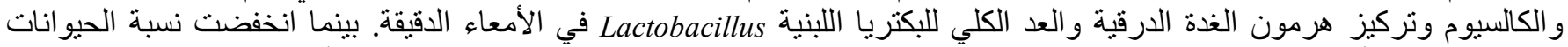

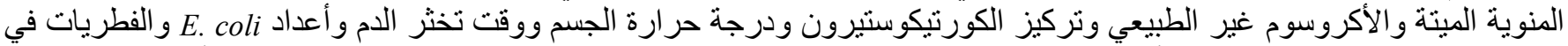

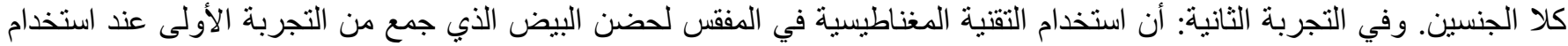




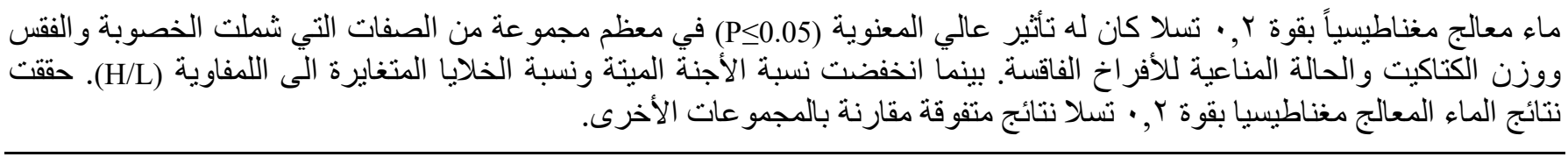

\section{Introduction}

Water is the most crucial nutrient for poultry, also it softens food and carries it through the body, assists in digestion and absorption, cools the body as it evaporates through the bird's lungs and air sacs. Water assist lubricate joints, is a major component of blood, remove wastes and a necessary medium for many chemical reactions that help form meat and eggs production (1). Water is needed to transport compounds via the blood, maintain cellular structural integrity, regulate temperature (2). Magnetic water (anti-scale magnetic) treatment define as a method of supposedly reduction the effects of hard water by passing it through a magnetic device, magnetized water is hexagonal, can activate and ionize water molecules to change its structure, it is effective in several chronic diseases including diabetes that caused by oxidative stress (3). In a magnetic field, magnetic force can break large water clusters into single molecules or smaller ones and increasing polarization effects of water molecules (4), also reduces the hydrogen-oxygen bond angle within the water molecule from 104 to 103 degrees, leading to better water absorption across cell membranes (5). Magnetic fields change osmotic processes in muscles, affect the permeability of the cellular membrane and disturb the hydration ability of animal tissues. The possible health effects of magnetic fields on reproduction and development including gametogenesis, fertilization, embryo-genesis and endocrine systems have been extensively studied (6). Treating water with magnetic fields simple regenerates the natural energy and balance that nature intended (7). Magnetic water reduces the water temperature, which regulates and turn down body temperature in hot climates due to excess the rate of digestion and absorption (8). Dramatically improvement of broiler breeder performance by producing more eggs, egg weight, hatchability, immunity, physiology and microbiological so survived longer when used magnetized water (9). Magnetized well water improved body weight and feed conversion rate of birds, besides renal and hepatic functions; it also increased production, quality and hatchability of eggs, levels of reproductive hormones (progesterone and estrogen), and the blood antioxidant status (10).

The aims of this paper lies in the following aspects: i) investigate the beneficial effect of magnetically treated water at levels 0.1 and 0.2 Tesla power field on the dramatically changes on physical and chemical characteristics of well water. ii) the importance of MTW uses in quail farm on productive and physiological traits under heat stress. iii) the benefit of using MTW during incubating eggs in hatchery by conducted MTW device on pipes that supplies water to humidity device inside incubator and hatchery and its effect on embryonic development, hatchability and maternal immunity statues. iv) calculated the economic profit of each bird to have knowledge the gross of this project.

\section{Materials and methods}

The two experiments were carried out in Grdarasha Poultry Houses and Hatchery of Animal Resources Department, College of Agriculture, Salahuddin UniversityErbil/ Iraq.

Experiment 1: Using magnetic technology in local quail house on production performances and physiological traits: 405 local adult quails (Coturnix coturnix) with average weight $219 \pm 10 \mathrm{~g}$ for females and $198 \pm 10 \mathrm{~g}$ for males at age $22 \mathrm{wk}$. (270 females and 135 males) were distributed into three groups, each group contains nine replicates as following: group 1: Well water (WW) as Control, group 2: MTW 0.1 T (1000 gauss), group 3: MTW 0.2 Tesla (2000 gauss). The quail birds were reared in special cages $65 \mathrm{~cm} \times 60 \mathrm{~cm} \times 50 \mathrm{~cm}$ length, width and height respectively. Three sets of cages used one for WW group, 2 cages one for $0.1 \mathrm{~T}$ MT and the other for $0.2 \mathrm{~T}$ MTW group, each set included 9 cages designed for quails. The farm temperature was controlled under $35-37^{\circ} \mathrm{C}$ afternoon and $28-30 \mathrm{C}^{\circ}$ at night and early morning. In addition, the hens were exposed to a 16-h photoperiod for a period of 70 days. Feed and water were available to permit ad libitum consumption.

The production ration of quail was formulated according to the NRC (11), the basal composition of the diet contains (55.36 wheat, 6.50 wheat bran, 7.00 wheat flour, 18.50 soya bean meal, 2.50 Bred-mix, 2.50 soybean oil, 7.30 limestone, 0.05 salt, 0.03 methionine, 0.03 lysine, 0.03 anti-toxin, 0.05 multi-minerals, 0.10 multi-vitamins, 0.05 enzymes) $\%$, the chemical analysis of these ingredients are $(18.0 \%$ Crude protein, $4.45 \%$ fat, $2802 \mathrm{ME}$. Kcal $/ \mathrm{kg}$, $155.82 \mathrm{C} / \mathrm{P}$ ratio, 3.91 Crude fibers, $3.05 \% \mathrm{Ca}, 0.38 \%$ available $\mathrm{P}, 0.81 \%$ lysine, $0.42 \%$ methionine).

Bipolar or dipole magnetically device or called magnetron about 0.1 and 0.2 Tesla in a diameter $1 \mathrm{inch}$, manufactured by the UAE magnetic technology company. The direction of the passage of water from the north pole towards the south pole. The magnet device connected by pipes that provide water to the cages of quails.

At end of the study females egg production (H.D \%), feed intake, feed conversion ratio (FCR), water 
consumption, egg weight, eggshell strength was calculated and plasma estrogen hormone concentration was measured by RIA.

In males: Feed and water were withheld from the males at least $6 \mathrm{~h}$ prior to semen collection, in order to minimize contamination of the semen with dropping then semen samples were collected according to (12). Semen was collected by stimulation of the male with a teaser female. The foam was removed from the cloacal gland by delicate squeezing of the gland by thumb. Semen was collected twice a week from each male by using a small glass collector fitted, with rubber tubing and a mouthpiece, into a small calibrated tube enabling measurement of ejaculate volume exact to $10 \mu \mathrm{l}$. The fresh collected semen was evaluated: immediately after collection in each ejaculate such as; semen ejaculates volume, sperm concentration, Live and normal morphology sperm, semen quality factor, dead sperm percentage, seminal plasma testosterone hormone conc. were measured by Radio immunoassay (RIA) using kits purchased from Biochem Immuno Systems.

At the end of study, blood samples were collected from the brachial vein of 15 birds of each group using EDTA tubes, the total $\mathrm{RBC}\left(10^{6} / \mathrm{ml}^{-1}\right.$ blood) were determined by using Natt and Herrick diluting solution (13). Differential of heterophil and lymphocyte made on slides stained with Wright-Giemsa and observed in an optical microscope $(100 \mathrm{x})$ to determined $\mathrm{H} / \mathrm{L}$ ratio. Blood and plasma viscosity were measured by Ostwald-viscom. Minerals $\mathrm{Fe}$ and $\mathrm{Cu}$ $(\mu \mathrm{g} / \mathrm{ml})$ and $\mathrm{Ca}(\mathrm{mg} / \mathrm{ml})$ were measured by total reflection $\mathrm{X}$ - ray fluorescence spectrometer (TXRF) technique. Body temperature was measured in both sexes by inserting mercury thermometer in rectal. Plasma thyroxin and corticosterone concentration were measured by RIA. Jejunum contents were cautiously kept in sterile petri dishes at $20^{\circ} \mathrm{C}$ until analyses in the laboratory. One gram of each homogenized sample was collected and transferred into 10 $\mathrm{ml}$ sterile saline solution for dilution. The next step, each sample was spread on selective agar plates as follows. The Nutrient and McConkey agars were utilized for E. coli and fungi as medium cultures (14) and MRS agar medium was used for Lactobacillus bacteria. The number of fungi and bacterial colonies were computed after incubation at $37^{\circ} \mathrm{C}$ for 48 hours. After that, the result numbers were converted to $\log 10$ per $\mathrm{ml}$ before statistical analysis.

Experiment 2: Using magnetic technology in hatching eggs on hatchability traits: A total of 1250 fertile eggs in average weight $11.85 \pm 0.3 \mathrm{~g}$ was collected from experiment 1 then incubated in the hatchery. Magnet device was connected by pipes that supply water to humidity device inside incubator and hatchery. The groups of this experiment included: i) Well water (WW) set 250 eggs. ii) 250 eggs collected from quail exposed to $0.1 \mathrm{~T}$ MTW and in the hatchery. iii) 250 eggs collected from quail exposed to $0.1 \mathrm{~T}$ MTW but not in the hatchery. iv) 250 eggs collected from quail treated with 0.2 Tesla MTW and in the hatchery. v) 250 eggs collected from quail treated with 0.2 Tesla MTW but not in the hatchery.

Eggs were set for $14 \mathrm{~d}$ in an incubator at $99.5^{\circ} \mathrm{F}$ $\left(37.5^{\circ} \mathrm{C}\right)$ and $55 \%$ relative humidity, then transferred to the hatchery for $3 \mathrm{~d}$ at $98.6^{\circ} \mathrm{F}\left(37^{\circ} \mathrm{C}\right)$ and $65 \%$ relative humidity until the end of $\mathrm{d} 17$ of incubation. At the end of hatching all live and dead chicks were counted, the percentages of (fertility, hatching of fertile and total eggs, dead embryos, culled chicks) and chicks weight. 12 chicks in each group were sacrificed for determining $\mathrm{H} / \mathrm{L}$ ratio its prepared smears stained by Wright-Giemsa. Serum was harvested after blood centrifuged from 1d old chicks to measure the antibody titer of Newcastle Disease (HI), Infectious Bursal Disease (IBD) or Gumboro and Infectious Bronchitis Viral (IBV) were measured by ELISA.

All data were analyzed by using CRD (Complete Randomize Design) by SAS (15), as per variance, significant differences among treatment means were determined by Duncan's multiple range tests at level 0.05 (16).

\section{Results}

Three samples of water which supplied to the quail groups were analyzed in laboratories of health directory in Erbil, The physic-chemical analysis of water observed 0.1 and $0.2 \mathrm{~T}$ MTW were significantly $(\mathrm{P} \leq 0.05)$ higher in $\mathrm{pH}$ value, consistency, dissolved oxygen and $\mathrm{Ca}^{+2}$, also $0.2 \mathrm{~T}$ MTW achieved superiorly in electrical conductivity (EC) and solubility. Whereas, 0.1 and $0.2 \mathrm{~T}$ MTW were significantly $(\mathrm{P} \leq 0.05)$ lower or improved in turbidity, hardness, alkalinity, total dissolved solids (TDS), $\mathrm{CO}_{2}$, soluble chloride concentration and $\mathrm{NO}_{3}{ }^{-}$also $0.2 \mathrm{~T}$ MTW decreased in evaporating temperature (ET), Surface tension (ST).

Results in table 2 revealed that quail females when drank magnetically treated water ( 0.1 and $0.2 \mathrm{~T}$ MTW) were significantly $(\mathrm{P} \leq 0.05)$ higher in egg Production (HD) $\%$, shell strength and estrogen concentration, also egg weight and water consumption were higher in $0.2 \mathrm{~T}$ MTW only compared with the quails in control group. Also, feed conversion ratio (FCR) improved in 0.2 T MTW. However, no significant differences among the quails of groups in feed intake.

Table 3 exposed that quail males served drinking $0.2 \mathrm{~T}$ MTW were significantly $(\mathrm{P} \leq 0.05)$ higher in ejaculate volume, sperm concentration, mass motility $\%$, live and normal morphology sperm $\%$ and semen quality factors. Also increased in quantity of foam, individual motility \% and testosterone hormone concentration in both 0.1 and 0.2 T MTW. While, dead sperm\% significantly $(\mathrm{P} \leq 0.05)$ lower 
in 0.1 and $0.2 \mathrm{~T}$ MTW and abnormal acrosome $\%$ in $0.2 \mathrm{~T}$ MTW compared with WW group.

Table 4 showed that quails when served drinking MTW were significantly $(\mathrm{P} \leq 0.05)$ higher in Total $\mathrm{RBC}$, plasma viscosity, blood iron $(\mathrm{Fe})$ and thyroxin hormone concentration in group $0.2 \mathrm{~T}$ MTW male and female, thrombocytes in groups 0.1 and $0.2 \mathrm{~T}$ MTW male and $0.2 \mathrm{~T}$ MTW female, blood viscosity, $\mathrm{pH}$ and $\mathrm{Cu}$ in groups presented 0.1 and $0.2 \mathrm{~T}$ MTW of both sexes, blood calcium $\left(\mathrm{Ca}^{+2}\right)$ in groups 0.1 and $0.2 \mathrm{~T}$ MTW in females only. However, MTW recorded significantly $(\mathrm{P} \leq 0.05)$ lower or improvement in corticosterone hormone concentration and body temperature in group $0.2 \mathrm{~T} \mathrm{MTW}$, also noticed reduction in blood clotting time in group $0.2 \mathrm{~T}$ MTW of males and in the groups 0.1 and $0.2 \mathrm{~T}$ MTW of female compared with control groups in both sexes.

Table 5 the using of magnetically treated water in quail house in both groups 0.1 and $0.2 \mathrm{~T}$ were significantly $(\mathrm{P} \leq 0.05)$ increase-ed the total count of Lactobacillus in small intestine, while $E$. Coli and fungi counts were significantly $(\mathrm{P} \leq 0.05)$ lower in 0.1 and $0.2 \mathrm{~T}$ MTW compared with the WW group in both males and females.

Table 1: Effect of magnetic technology on physical and chemical analysis of water

\begin{tabular}{|c|c|c|c|}
\hline \multirow{2}{*}{ Physical analysis } & \multirow{2}{*}{ WW } & \multicolumn{2}{|c|}{ MTW } \\
\hline & & $0.1 \mathrm{~T}$ & $0.2 \mathrm{~T}$ \\
\hline $\mathrm{pH}$ value & $7.34^{b}$ & $8.03^{\mathrm{a}}$ & $8.15^{\mathrm{a}}$ \\
\hline $\mathrm{EC}(\mu \mathrm{s} / \mathrm{cm})$ & $489^{\mathrm{a}}$ & $503^{a b}$ & $512^{\mathrm{a}}$ \\
\hline Turbidity (NTU)* & $528^{\mathrm{a}}$ & $405^{\mathrm{b}}$ & $347^{\mathrm{c}}$ \\
\hline Hardness (mg/L) & $154^{\mathrm{a}}$ & $115^{\mathrm{b}}$ & $92^{\mathrm{c}}$ \\
\hline ET (g/hour) & $0.688^{\mathrm{a}}$ & $0.629^{\mathrm{ab}}$ & $0.573^{\mathrm{b}}$ \\
\hline ST (Dyn/cm) & $69.91^{\mathrm{a}}$ & $69.03^{\mathrm{a}}$ & $67.33^{b}$ \\
\hline Consistency $(\mathrm{g} / \mathrm{ml})$ & $0.9936^{\mathrm{b}}$ & $1.0008^{\mathrm{a}}$ & $1.0013^{\mathrm{a}}$ \\
\hline Solubility $(\mathrm{g} / 10 \mathrm{ml})$ & $2.93^{\mathrm{b}}$ & $3.11^{\mathrm{ab}}$ & $3.28^{\mathrm{a}}$ \\
\hline Viscosity (centipoise) & $0.726^{\mathrm{a}}$ & $0.704^{\mathrm{ab}}$ & $0.688^{b}$ \\
\hline Salinity $(\%)$ & $29.45^{\mathrm{a}}$ & $28.72^{\mathrm{ab}}$ & $28.31^{\mathrm{b}}$ \\
\hline Alkalinity (ppm) & $130.1^{\mathrm{a}}$ & $119.6^{\mathrm{b}}$ & $113.6^{\mathrm{c}}$ \\
\hline \multicolumn{4}{|l|}{ Chemical analysis } \\
\hline TDS (mg/L) & $422^{\mathrm{a}}$ & $392^{b}$ & $312^{\mathrm{c}}$ \\
\hline Dissolved oxygen \% & $36.18^{c}$ & $40.11^{b}$ & $46.39^{\mathrm{a}}$ \\
\hline $\mathrm{CO}_{2}(\mathrm{ppm})$ & $10.73^{\mathrm{a}}$ & $6.02^{\mathrm{b}}$ & $4.37^{\mathrm{c}}$ \\
\hline $\begin{array}{l}\text { Soluble Chloride } \\
\text { concentration (ppm) }\end{array}$ & $57.84^{\mathrm{a}}$ & $47.59^{b}$ & $40.69^{\mathrm{c}}$ \\
\hline $\mathrm{Ca}^{+2}(\mathrm{mg} / \mathrm{L})$ & $17.92^{\mathrm{c}}$ & $24.73^{b}$ & $28.96^{\mathrm{a}}$ \\
\hline $\mathrm{NO}_{3}^{-}(\mathrm{mg} / \mathrm{L})$ & $3.59^{\mathrm{a}}$ & $2.06^{\mathrm{b}}$ & $1.55^{\mathrm{b}}$ \\
\hline
\end{tabular}

WW: Well water (control), MTW: magnetically treated water, NTU: nephelometric turbidity units, the same superscripts within rows means non-significant, ${ }^{\mathrm{a}-\mathrm{c}}$ Means within rows with different superscripts differ significantly at $(\mathrm{P} \leq 0.05)$.
Table 2: Effect of magnetically treated water on some productive parameters and estrogen concentration of local quail females

\begin{tabular}{lcccc}
\hline \multirow{2}{*}{ Characteristics } & \multirow{2}{*}{ WW } & \multicolumn{2}{c}{ MTW } & \multirow{2}{*}{ SEM } \\
\cline { 3 - 4 } & & $0.1 \mathrm{~T}$ & $0.2 \mathrm{~T}$ & \\
\hline Egg Production\% & $80.67^{\mathrm{c}}$ & $87.22^{\mathrm{b}}$ & $92.58^{\mathrm{a}}$ & 2.45 \\
Feed Intake (g) & $2433.5^{\mathrm{a}}$ & $2441.2^{\mathrm{a}}$ & $2330.7^{\mathrm{a}}$ & 112 \\
FCR (g feed/g egg) & $2.55^{\mathrm{a}}$ & $2.27^{\mathrm{a}}$ & $1.90^{\mathrm{b}}$ & 0.18 \\
Water consumption & $70.13^{\mathrm{b}}$ & $73.40^{\mathrm{ab}}$ & $79.22^{\mathrm{a}}$ & 3.19 \\
(ml/bird/d) & $11.83^{\mathrm{b}}$ & $12.33^{\mathrm{b}}$ & $13.25^{\mathrm{a}}$ & 0.47 \\
$\begin{array}{l}\text { Egg Weight (g) } \\
\text { Shell Strength }\end{array}$ & $633^{\mathrm{b}}$ & $691^{\mathrm{a}}$ & $728^{\mathrm{a}}$ & 36.8 \\
(g/cm $^{2}$ ) & $0.533^{\mathrm{c}}$ & $0.810^{\mathrm{b}}$ & $0.903^{\mathrm{a}}$ & 0.104 \\
\hline Estrogen (ng/ml) & &
\end{tabular}

WW: Well water (control), MTW: magnetically treated water, FCR: feed conversion ratio, the same superscripts within rows means non-significant, ${ }^{\mathrm{a}-\mathrm{c}}$ Means within rows with different superscripts differ significantly at $(\mathrm{P} \leq 0.05)$.

Table 3: Effect of magnetically treated water on sperm quality of local quail males

\begin{tabular}{|c|c|c|c|c|}
\hline \multirow{2}{*}{ Characteristics } & \multirow{2}{*}{ WW } & \multicolumn{2}{|c|}{ MTW } & \multirow{2}{*}{ SEM } \\
\hline & & $0.1 \mathrm{~T}$ & $0.2 \mathrm{~T}$ & \\
\hline $\begin{array}{l}\text { quantity of foam } \\
\text { (mg) }\end{array}$ & 4.11 & $104.9^{\mathrm{a}}$ & $89.5^{\mathrm{b}}$ & $72.0^{\mathrm{c}}$ \\
\hline $\begin{array}{l}\text { Ejaculate volume } \\
(\mu \mathrm{L})\end{array}$ & 4.85 & $53.1^{\mathrm{a}}$ & $37.9^{b}$ & $34.3^{\mathrm{b}}$ \\
\hline $\begin{array}{l}\text { Sperm concen. } \\
\left(10^{6} / \mathrm{ml}^{-1}\right)\end{array}$ & 47.9 & $825.3^{\mathrm{a}}$ & $690.3^{b}$ & $652.5^{b}$ \\
\hline Mass Motility \% & 3.16 & $94.05^{\mathrm{a}}$ & $83.58^{b}$ & $80.92^{b}$ \\
\hline $\begin{array}{l}\text { Individual Motility } \\
\%\end{array}$ & 2.97 & $97.10^{\mathrm{a}}$ & $88.17^{\mathrm{b}}$ & $83.93^{\mathrm{c}}$ \\
\hline $\begin{array}{l}\text { Live and normal } \\
\text { sperm } \%\end{array}$ & 1.88 & $83.33^{\mathrm{a}}$ & $68.75^{\mathrm{b}}$ & \\
\hline $\begin{array}{l}\text { Semen quality } \\
\text { factor }\end{array}$ & 1.04 & $36.52^{\mathrm{a}}$ & $17.99^{b}$ & $15.02^{b}$ \\
\hline sperm \% & 0.75 & $5.43^{\mathrm{c}}$ & $11.55^{\mathrm{b}}$ & $15.00^{\mathrm{a}}$ \\
\hline 1 sperm $\%$ & 1.02 & $8.00^{\mathrm{b}}$ & $15.10^{\mathrm{a}}$ & $13.90^{\mathrm{a}}$ \\
\hline $\begin{array}{l}\text { Abnormal } \\
\text { acrosome } \%\end{array}$ & 0.306 & $3.24^{\mathrm{a}}$ & $4.60^{\mathrm{a}}$ & $3.99^{\mathrm{a}}$ \\
\hline $\begin{array}{l}\text { Testosterone } \\
\text { hormone (ng/ml) }\end{array}$ & 0.218 & $5.29^{\mathrm{a}}$ & $4.33^{b}$ & $3.67^{\mathrm{c}}$ \\
\hline
\end{tabular}

WW: Well water (control), MTW: magnetically treated water, FCR: feed conversion ratio, ${ }^{\mathrm{a}-\mathrm{c}}$ Means within rows with different superscripts differ significantly at $(\mathrm{P} \leq 0.05)$.

\section{Experiment 2}

Table 6 showed the results of magnetically treated water on hatchability. The eggs at the end of $17^{\text {th }}$ day which treated and untreated groups with $0.2 \mathrm{~T}$ MTW in hatchery were significantly $(\mathrm{P} \leq 0.05)$ higher in fertility $\%$, 
hatchability of fertile and total egg \% and hatched chicks weight, also treated group with $0.1 \mathrm{~T}$ MTW had increased in hatchability of fertile and total egg \% compared with WW group. However significantly $(\mathrm{P} \leq 0.05)$ lower in total dead embryo and culled chicks percentage in $0.2 \mathrm{~T}$ MTW and $0.1 \mathrm{~T}$ MTW with the treated group compared with WW group.
The antibodies titer of hatched chicks by ELISA shows significantly $(\mathrm{P} \leq 0.05)$ higher of $\mathrm{ND}, \mathrm{IBD}$ and IBV in $0.2 \mathrm{~T}$ MTW in treated and untreated and 0.1 T MTW in treated group compared with $0.1 \mathrm{~T}$ MTW in untreated and WW groups. While, $\mathrm{H} / \mathrm{L}$ ratio were significantly $(\mathrm{P} \leq 0.05)$ lower or improved in all groups of MTW as compared with WW group (Table 7).

Table 4: Effect of magnetically treated water on blood and plasma characteristics of local quail males and females

\begin{tabular}{|c|c|c|c|c|c|c|c|c|}
\hline \multirow{3}{*}{$\begin{array}{l}\text { Characteristics } \\
\text { Sex }\end{array}$} & \multirow{2}{*}{\multicolumn{2}{|c|}{ WW }} & \multicolumn{4}{|c|}{ MTW } & \multirow{2}{*}{\multicolumn{2}{|c|}{ SEM }} \\
\hline & & & \multicolumn{2}{|c|}{$0.1 \mathrm{~T}$} & \multicolumn{2}{|c|}{$0.2 \mathrm{~T}$} & & \\
\hline & $\mathrm{M}$ & $\mathrm{F}$ & $\mathrm{M}$ & $\mathrm{F}$ & $\mathrm{M}$ & $\mathrm{F}$ & $\mathrm{M}$ & $\mathrm{F}$ \\
\hline Total RBC $\left(10^{6} / \mathrm{ml}^{-1}\right.$ blood $)$ & $4.27^{b}$ & $4.02^{b}$ & $4.85^{\mathrm{ab}}$ & $4.39^{b}$ & $5.09^{\mathrm{a}}$ & $4.88^{\mathrm{a}}$ & 0.612 & 0.417 \\
\hline Thrombocyte $\left(10^{3} / \mathrm{ml}^{-1}\right.$ blood $)$ & $22.48^{\mathrm{c}}$ & $22.10^{\mathrm{b}}$ & $23.05^{\mathrm{b}}$ & $22,83^{\mathrm{b}}$ & $25.79^{\mathrm{a}}$ & $25.13^{\mathrm{a}}$ & 1.33 & 1.45 \\
\hline Blood viscosity (centipoise) & $2.31^{\mathrm{b}}$ & $2.18^{\mathrm{b}}$ & $2.66^{\mathrm{a}}$ & $2.57^{\mathrm{a}}$ & $2.84^{\mathrm{a}}$ & $2.69^{\mathrm{a}}$ & 0.26 & 0.22 \\
\hline Plasma viscosity (centipoise) & $0.94^{\mathrm{b}}$ & $1.16^{\mathrm{b}}$ & $1.08^{\mathrm{ab}}$ & $1.38^{\mathrm{ab}}$ & $1.39^{\mathrm{a}}$ & $1.53^{\mathrm{a}}$ & 0.11 & 0.15 \\
\hline Blood clotting time $(\mathrm{sec})$ & $110^{\mathrm{a}}$ & $117^{\mathrm{a}}$ & $102^{\mathrm{ab}}$ & $105^{\mathrm{b}}$ & $94^{\mathrm{b}}$ & $97^{\mathrm{b}}$ & 4.25 & 5.11 \\
\hline Blood $\mathrm{pH}$ & $7.16^{\mathrm{b}}$ & $7.28^{\mathrm{b}}$ & $7.43^{\mathrm{a}}$ & $7.65^{\mathrm{a}}$ & $7.62^{\mathrm{a}}$ & $7.83^{\mathrm{a}}$ & 0.703 & 0.854 \\
\hline Blood Fe $(\mu \mathrm{g} / \mathrm{ml})$ & $0.849^{b}$ & $0.826^{\mathrm{b}}$ & $1.272^{\mathrm{ab}}$ & $1.035^{\mathrm{ab}}$ & $1.617^{\mathrm{a}}$ & $1.419^{\mathrm{a}}$ & 0.134 & 0.152 \\
\hline Blood $\mathrm{Cu}(\mu \mathrm{g} / \mathrm{ml})$ & $0.289^{\mathrm{b}}$ & $0.290^{\mathrm{b}}$ & $0.390^{\mathrm{a}}$ & $0.368^{\mathrm{a}}$ & $0.475^{\mathrm{a}}$ & $0.453^{\mathrm{a}}$ & 0.078 & 0.054 \\
\hline Blood $\mathrm{Ca}^{+2}(\mathrm{mg} / \mathrm{ml})$ & $9.57^{\mathrm{a}}$ & $15.03^{\mathrm{b}}$ & $10.13^{\mathrm{a}}$ & $17.69^{\mathrm{a}}$ & $10.42^{\mathrm{a}}$ & $19.04^{\mathrm{a}}$ & 0.820 & 0.692 \\
\hline Corticosterone $(\mathrm{ng} / \mathrm{ml})$ & $2.035^{\mathrm{a}}$ & $1.675^{\mathrm{a}}$ & $1.739^{\mathrm{ab}}$ & $1.245^{\mathrm{ab}}$ & $1.398^{\mathrm{b}}$ & $0.917^{\mathrm{b}}$ & 0.105 & 0.094 \\
\hline Thyroxin (T4) (ng/ml) & $1.157^{\mathrm{b}}$ & $1.209^{\mathrm{b}}$ & $1.400^{\mathrm{ab}}$ & $1.715^{b}$ & $1.606^{\mathrm{a}}$ & $1.925^{\mathrm{a}}$ & 0.128 & 0.203 \\
\hline Body Temperature $\left({ }^{\circ} \mathrm{C}\right)$ & $41.42^{\mathrm{a}}$ & $41.67^{\mathrm{a}}$ & $40.92^{\mathrm{ab}}$ & $41.08^{\mathrm{ab}}$ & $39.97^{\mathrm{b}}$ & $40.05^{b}$ & 1.33 & 0.93 \\
\hline
\end{tabular}

WW: Well water (control), MTW: magnetically treated water. The same superscripts within rows means non-significant, ${ }^{\mathrm{a}-\mathrm{c}}$ Means within rows with different superscripts differ significantly at $(\mathrm{P} \leq 0.05)$.

Table 5: Effect of magnetically treated water on bacterial and fungi count $\left(\mathrm{cfu} \times 10^{5} / \mathrm{g}\right)$ content of small intestine of local quail

\begin{tabular}{|c|c|c|c|c|c|c|c|c|}
\hline \multirow{3}{*}{$\begin{array}{l}\text { Characteristics } \\
\text { Sex }\end{array}$} & \multirow{2}{*}{\multicolumn{2}{|c|}{ WW }} & \multicolumn{4}{|c|}{ MTW } & \multirow{2}{*}{\multicolumn{2}{|c|}{ SEM }} \\
\hline & & & \multicolumn{2}{|c|}{$0.1 \mathrm{~T}$} & \multicolumn{2}{|c|}{$0.2 \mathrm{~T}$} & & \\
\hline & $\mathrm{M}$ & $\mathrm{F}$ & $\mathrm{M}$ & $\mathrm{F}$ & $\mathrm{M}$ & $\mathrm{F}$ & $\mathrm{M}$ & $\mathrm{F}$ \\
\hline Lactobacillus & $5.52^{b}$ & $5.13^{b}$ & $7.75^{\mathrm{a}}$ & $7.12^{\mathrm{a}}$ & $8.47^{\mathrm{a}}$ & $8.25^{\mathrm{a}}$ & 0.906 & 0.762 \\
\hline E. coli & $8.22^{\mathrm{a}}$ & $8,46^{\mathrm{a}}$ & $5.94^{b}$ & $6.35^{\mathrm{b}}$ & $5.65^{\mathrm{b}}$ & $5.21^{\mathrm{b}}$ & 0.495 & 0.602 \\
\hline Fungi & $7.39^{\mathrm{a}}$ & $7.63^{a}$ & $3.86^{\mathrm{b}}$ & $4,25^{\mathrm{b}}$ & $4.74^{b}$ & $5.03^{b}$ & 0.477 & 0.510 \\
\hline
\end{tabular}

WW: Well water (control), MTW: magnetically treated water, cfu: colony forming unit, ${ }^{\mathrm{a}-\mathrm{c}}$ Means within rows with different superscripts differ significantly at $(\mathrm{P} \leq 0.05)$.

Table 6: Effect of magnetically treated water on hatchability characteristics of local quail incubated eggs

\begin{tabular}{lcccccc}
\hline \multirow{2}{*}{ Characteristics } & \multirow{2}{*}{ WW } & \multicolumn{2}{c}{ MTW $(0.1 \mathrm{~T})$} & \multicolumn{2}{c}{ MTW $(0.2 \mathrm{~T})$} & \multirow{2}{*}{ SEM } \\
\cline { 3 - 5 } & & treated & non & treated & non & \\
\hline Fertility \% & $80.77^{\mathrm{b}}$ & $83.25^{\mathrm{b}}$ & $83.45^{\mathrm{b}}$ & $93.70^{\mathrm{a}}$ & $90.51^{\mathrm{a}}$ & 1.83 \\
Hatchability of fertile egg \% & $77.09^{\mathrm{bc}}$ & $81.05^{\mathrm{b}}$ & $74.16^{\mathrm{c}}$ & $89.62^{\mathrm{a}}$ & $85.36^{\mathrm{ab}}$ & 1.08 \\
Hatchability of total egg \% & $70.76^{\mathrm{c}}$ & $78.30^{\mathrm{b}}$ & $71.31^{\mathrm{c}}$ & $88.62^{\mathrm{a}}$ & $83.81^{\mathrm{ab}}$ & 1.22 \\
Total dead embryos $(1-17) \mathrm{d} \%$ & $14.56^{\mathrm{a}}$ & $13.00^{\mathrm{b}}$ & $13.70^{\mathrm{ab}}$ & $5.30^{\mathrm{c}}$ & $7.99^{\mathrm{c}}$ & 0.031 \\
Culled chicks \% & $4^{\mathrm{a}}$ & $2.67^{\mathrm{a}}$ & $3.75^{\mathrm{ab}}$ & $1.00^{\mathrm{c}}$ & $1.50^{\mathrm{c}}$ & 0.130 \\
Hatched chicks weight $(\mathrm{g})$ & $8.15^{\mathrm{b}}$ & $8.23^{\mathrm{b}}$ & $8.04^{\mathrm{b}}$ & $9.05^{\mathrm{a}}$ & $8.90^{\mathrm{a}}$ & 0.55 \\
\hline
\end{tabular}

The same superscripts within rows means non-significant. ${ }^{a-c}$ Means within rows with different superscripts differ significantly at $(\mathrm{P} \leq 0.05)$. 
Table 7: Effect of magnetically treated water on immunological ELISA titer ( $\mathrm{ng} / \mathrm{ml})$ against some diseases of hatched quail chicks

\begin{tabular}{|c|c|c|c|c|c|c|}
\hline \multirow{2}{*}{ Characteristics } & \multirow{2}{*}{ WW } & \multicolumn{2}{|c|}{ MTW $(0.1 \mathrm{~T})$} & \multicolumn{2}{|c|}{$\operatorname{MTW}(0,2 \mathrm{~T})$} & \multirow{2}{*}{ SEM } \\
\hline & & treated & non & treated & non & \\
\hline ND & $5280^{c}$ & $6709^{b}$ & $5437^{\mathrm{c}}$ & $8011^{\mathrm{a}}$ & $7183^{\mathrm{ab}}$ & 132 \\
\hline IBD & $2785^{c}$ & $3780^{\mathrm{ab}}$ & $3167^{\mathrm{bc}}$ & $4307^{\mathrm{a}}$ & $4121^{a}$ & 122 \\
\hline IBV & $1780^{c}$ & $1966^{b}$ & $1839^{\mathrm{bc}}$ & $2417^{\mathrm{a}}$ & $2295^{\mathrm{a}}$ & 98.6 \\
\hline $\mathrm{H} / \mathrm{L}$ ratio & $0.711^{\mathrm{a}}$ & $0.503^{b}$ & $0.579^{\mathrm{b}}$ & $0.391^{\mathrm{c}}$ & $0.422^{b c}$ & 0.103 \\
\hline
\end{tabular}

${ }^{\mathrm{a}-\mathrm{c}}$ Means within rows with different superscripts differ significantly at $(\mathrm{P} \leq 0.05)$. ND: Newcastle disease; IBD: Gumboro disease, IBV: Infectious bronchitis viral disease.

Table 8 declare the using of 0.1 and 0.2 T MTW in quail house during the laying period and in humidity of the hatchery significantly $(\mathrm{P} \leq 0.05)$ raise the economical profit compared with the WW the control group.

Table 8: Effect of magnetically treated water on economical profit (outcome) (\$/ bird)

\begin{tabular}{lcccc}
\hline \multirow{2}{*}{ Characteristics } & \multirow{2}{*}{ WW } & \multicolumn{2}{c}{ MTW } & \multirow{2}{*}{ SEM } \\
\cline { 3 - 4 } & & $0.1 \mathrm{~T}$ & $0.2 \mathrm{~T}$ & \\
\hline EP-laying period & $1.628^{\mathrm{b}}$ & $1.818^{\mathrm{ab}}$ & $2.175^{\mathrm{a}}$ & 0.176 \\
EP-hatching period & $0.433^{\mathrm{c}}$ & $0.545^{\mathrm{b}}$ & $0.671^{\mathrm{a}}$ & 0.1 .33 \\
Total EP & $2.061^{\mathrm{c}}$ & $2.363^{\mathrm{b}}$ & $2.846^{\mathrm{a}}$ & 0.210 \\
\hline
\end{tabular}

${ }^{\mathrm{a}-\mathrm{c}}$ Means within rows with different superscripts differ significantly at $(\mathrm{P} \leq 0.05)$. EP: Economic profit.

\section{Discussions}

Using application of magnetic technology in local quail exposed to heat stress, improved water quality by passing water through a magnetic device. Data in table 1 exist to considerate changes in water physical characteristics: $\mathrm{pH}$ value $(7.34,8.03,8.15)$, electrical conductivity (489, $503,5012 \mu \mathrm{s} / \mathrm{cm})$, turbidity $(528,405,347 \mathrm{NTU})$, hardness $(154,115,92 \mathrm{mg} / \mathrm{L})$, evaporating temperature $(0.688$, $0.629,0.573 \mathrm{~g} / \mathrm{h}$ ), surface tension $(69.91,69.03 .67 .33$ Dyn/cm), consistency $(0.9936,1.0008,1.0013 \mathrm{~g} / \mathrm{ml})$, solubility $(2.93,3.11,3.28 \mathrm{~g} / 10 \mathrm{ml})$, viscosity $(0.726,0.704$, 0.688 centipoise), salinity $(29.45,28.72,28.31 \%)$, refractive Index $(1.315,1.332,1.337$, alkalinity $(130.1,119.6,113,6$ ppm) and chemical characteristics: the total dissolved solids (422, 392,312 $\mathrm{mg} / \mathrm{L})$, dissolved oxygen $(36.18,40.11$, $46.39 \%), \mathrm{CO}_{2}(10.73,6.02,4.37 \mathrm{ppm})$, soluble chloride (57.84, 47.59, 40.69 ppm), $\mathrm{Ca}^{+2}(17.92,24.73,28.96 \mathrm{mg} / \mathrm{L})$, $\mathrm{NO}_{3}^{-}(3.59,2.06,1.55 \mathrm{mg} / \mathrm{L})$ of WW and $0.1 \mathrm{~T}$ MTW, 0.2 T MTW respectively.

The recovery of water physic-chemical quality by water passing through a magnetic field acquires a finer and more homogeneous structure (17), reduces the hydrogen-oxygen bond angle within the water molecule (5), due increase in water consumption $79.22 \mathrm{ml} /$ bird in the group $0.2 \mathrm{~T}$ MTW that led up to improvement in overall quail hens reproductive performance: egg production up to $92.58 \%$, egg weight $13.25 \mathrm{~g}$, and estrogen hormone concentration which increases water fluidity and ability to dissolve various constituents such as minerals and vitamins (18), leading to better water absorption across cell membranes. The recent results with the results of El-Hanoun et al. (10) exalted magnetic treatment of tap and well water improved water consumption, productive performance, such as number, weight and mass of eggs, feed conversion ratio of geese compared with groups without magnetically treated. For the same reasons above the male performance presented of ejaculate volume, sperm concentration, individual and mass motility, live and normal morphology sperm percentages, semen quality factor, quantity of foam and testosterone hormone concentration increased. While, decreased dead sperm and abnormal acrosome percentages. Consumption of MTW raised iron and copper concentrations in blood plasma led to increase in total number of erythrocyte and thrombocytes give rise to high blood and plasma viscosity which had a role in accelerating the process of blood clotting, which could be due to contain adequate of thromboplastin. In addition, MTW positively effect on thyroid gland by increasing the activity of the pituitary gland in the liberation thyrotrophic stimulation hormone for rise thyroxin hormone $\mathrm{T}_{4}$ concentration which enhanced metabolic rate. These results of blood ameliorate in agreement with Mustafa (9) when used MTW in drinking water in broiler.

Magnetic water causing increased production and transferred large amount of RBC (19) or perhaps causes increase in attract iron from the blood and then connect the blood in large quantities and thus increase number of RBCs (20), this is due to the possibility of magnetically treated water to increase the solubility of metal salts and nutrients, especially iron, which have been more willing to penetrate cells' membrane especially blood cells.

Magnetic field animated blood more flow than increases it, and distribute nutrients to the tissues much more effectively and quickly (21), so tissue and get more nourishment. The uplift of blood $\mathrm{Ca}^{+2}$ procures deposition 
of adequate calcium on egg shell which strengthens it in groups MTW in the female. The mostly studied ionic second messenger in cell magneto sensitivity is the cellular $\mathrm{Ca}^{+2}$. Respectively, many experiments have been performed on the effects of magnetic fields on the processes, involving calcium. Many of them can be affected by oscillating magnetic fields (22).

Using $0.2 \mathrm{~T}$ MTW in quail drinking act remarkable reduction in body temperature, it means regulating body temperature during heat stress from 41.42 to $39.97^{\circ} \mathrm{C}$ in the male and from 41.67 to $40.05{ }^{\circ} \mathrm{C}$ in the female, which depressed corticosterone hormone concentration from 2.035 to $1.398 \mathrm{ng} / \mathrm{ml}$ in male and from 1.675 to $0.917 \mathrm{ng} / \mathrm{ml}$ in female. Adams (8) elucidated MTW turn down body temperature in hot climates due excess the rate of digestion and absorption, Gross and Siegel (23) observed a significant positive correlation between the level of corticosterone and $\mathrm{H} / \mathrm{L}$. Passing water through magnetizer device $\mathrm{pH}$ become more alkaline 8.03 and 8.15 in water treated with 0.1 and $0.2 \mathrm{~T}$ respectively, which led to increase in blood $\mathrm{pH}$ from 7.16 (MTW) to 7.43 and 7.62 (0.1 and 0.2 T MTW) in male and from 7.28 (MTW) to 7.65 and 7.83 (0.1 and 0.2 T MTW) in female.

More alkalinity of MTW fulfilled increase of total count of Lactobacillus in small intestine, otherwise E. coli and fungi counts in both males and females. Exposure of water to the magnetic field caused marked changes in $\mathrm{pH}$ value, total dissolved $\mathrm{O}_{2}$ and solids, total hardness, electric conductivity, salinity, temperature of evaporation, mineral contents, organic matter and bacteria count (24), caused to destruct bacteria in water and it consequently reduces diseases by more than three times (25), other studies found exposing water to magnetic field increased the permeability of a cell membrane $(26,27)$. Which may lead to the loss of microbe viability (28). Also, magnetic field directly affected intracellular fluid and intracellular substances to activate enzymes inside the cells and to accelerate biochemical reactions in the body (29). Thus, it is possible that drinking magnetized water activates antioxidant enzymes in the body and reduces DNA damages. In biological systems there is a whole set of signaling mechanisms, based on various biochemical reactions (30). The increased of fertility and hatchability percentages, chicks weight and reduction in the dead embryo, also high development of maternal immunity (for ND, IBD and IBV diseases) of hatched chicks in the groups of quail drank MTW or received MTW in hatchery through humidity especially $0.2 \mathrm{~T}$ group. Embryogenesis is one of the most important developmental stages, and the biological value of adult organisms and breeding results are largely dependent on proper embryonic development. The embryo is especially sensitive to external factors that may have a detrimental effect on its development $(31,32)$. El-Hanoun et al. (10) evaluated magnetic treatment of tap and well water improved egg fertility, and egg hatchability, health status and biological performance, reproductive hormone levels, total antioxidant capacity of geese compared with groups without magnetically treated these effects could be due to increased mineral solubility, facilitating nutrient transfer across cell membranes thus, uptake and utilization (33-35). Blood heterophil to lymphocyte ratio (H/L) improved (recorded lower value) in the groups of MTW compared with well water group which increased $\mathrm{H} / \mathrm{L}$ ratio it is an indicator of stress, Gross and Siegel (23) observed a significant positive correlation between the level of corticosterone and $\mathrm{H} / \mathrm{L}$ ratio.

Finally, using MTW in quail drinking water and humidity of the hatchery increased egg production with rate $6.55,11.91 \%$ and hatching with rate $7.54,17.86 \%$ in the groups 0.1 and $0.2 \mathrm{~T}$ MTW respectively compared with the control group (WW), which had positively affected on economic profit.

\section{Conclusion}

The application of magnetically treated water (MTW) especially 0.2 Tesla (2000 gauss) had improved the physical and chemical characteristics of water and regulate body temperature, which gain high yield of egg about $11.91 \%$, improved feed conversion ratio, water consumption more eggshell strength in females and perfect semen quality in males. Besides promoting blood traits and better count of lactobacillus; it also increased embryonic development, fertility and hatchability of incubated eggs about $17.86 \%$ when used MTW in humidity device, levels of reproductive hormones (progesterone and estrogen), and the immunity status for neonatal hatched chicks, so resultant more benefit outcome of this study.

\section{References}

1. Blake JP, Joseph B Hess. Evaluating water quality for poultry. Alabama Cooperative Extension System ANR. 2001; p:1201.

2. Reuter R. Water is the most important nutrients. Nobel Foundation Agricultural Division, 2004. (http://www.nobel.org/Ag/ livestock/water important nutrient/).

3. Ma YL, Ren H, Ren S, Zhen EK, Hao G, Zhao YW. A study of the effect of magnetized water on enzyme activities by potentiometric enzyme electrode method. J Tongji Med Univ. 1992;12:193-6.

4. Pang XF, Deng B. Investigation of changes in properties of water under the action of a magnetic field. Sci China Ser G Phys Mech Astro. 2008;51 (11): 1-12.

5. Verma SS. Magnetic water treatment. Chem Business. 2011; 25:1316.

6. Kholodov YA. Influence of magnetic fields on biological objects. Am Rev Bicentennial. 1974;1776 -1976.

7. Ovchinnikova K, Pollack GH. Can water store charge?. Langmuir. 2009;25:542-547. http://dx.doi.org/10.1021/la802430k

8. Mustafa MGH. Effects of Magnetically Treated Water on Male Sexual Traits in Broiler Breeder During Hot Climate in Summer. Mesopotamia Journal of Agriculture. 2012, 40 (4): 347-350. 
9. Mustafa MA. Effect of magnetic technology of water treatment on productive and physiological performance of embryos, broiler breeders' and hatched chicks in different climates [PhD dissertation]. Baghdad: Agriculture at the University of Baghdad; 2007, p: 70-79.

10. El-Hanoun AM, Youssef AA, Mohammed A, Al-Harthi HI, Habiba MC. Magnetized drinking water improves productivity and blood parameters in geese. Rev Colomb Cienc Pecu. 2017;30:209-218. doi: 10.17533/udea.rccp.v30n3a04

11. NRC (National Research Council). Nutrient Requirements of poultry. $9^{\text {th }}$ ed. Washington: National Academy Press; 1994. P: 80- 105.

12. Bakst MR, Cecil HC. Techniques for semen evaluation, semen storage and fertility determination. Cornell: Poultry Science Association; 1997. Chapter 10, p: $175-195$. DOI: $10.5772 / 54918$

13. Natt MP, Herrick CA. A new blood diluents for counting the erythrocytes of the chicken. Poult Sci. 1952;31:735-738.

14. Harrigan WF, McCance MF. Laboratory methods. In: Food and dairy

a. microbiology. London: Academic Press; 1976.

15. SAS. User's guide for personal computer. New York: SAS Institute Inc; 2005. P: 1-48.

16. Duncan DB. Multiple range and multiple F-test. Biometrics. 1955;11:1-42.

17. Tkachenko Y, Semyonova N. Your way to health: magnetic water plus separate nutrition. In: Mysteries of magnetic energies. A collection of scientific works on the usage of magnetic energies in medical practice. Yuri PT editors. Sharjah: Printing and PublishingSharjah; 1995. Part 6, pp: 225-244.

18. Kronenberg KJ. Experimental evidence for effects of magnetic fields on moving water. Trans Magnetics. 1985;21:2059-2061.

19. Laycock DC. Pulsed magnetic field therapy and the physiotherapist. UK: Westville Associates and Consultants; 2007.

20. Rokicki R. Magnetic fields and electro-polished metallic implants. Medical Device and Diagnostic Industry; 2006, 28(3):116-123.

21. Ratchagar NP, Kumar VR. Effects of magnetic field on the red cell on nutritional transport in capillary-tissue exchange system. Inter $\mathrm{J}$ Mathemat Modeling Comput. 2014;4(1):1-15.

22. Voet D, Voet JG. Biochemistry. $3^{\text {rd }}$ ed. New York: John Wiley and Sons; 2004; Illustrated, 28 p. 1-1591.

23. Gross WB, Siegel HS. Evaluation of the heterophil/Lymphocyte ratio as measure of stress in chickens. Avian Dis. 1983;27:972-979.

24. Shaban AE, Azab EA. Biological effects of magnetic water on human and animals. Biomed Sci. 2017;3(4):78-85. doi: 10.11648/j.bs.20170304.12 https://www.preprints.org/manuscript/201711.0202/v1
26. Varkey AJ, Mgidi DD, Simiso KM. Decontamination of bacteria from water with moderate electric and magnetic fields. 2017;11:22-25. https://www.preprints.org/manuscript/201711.0202/v1.

27. Greig JD, Todd ECD, Bartleson C, Michaels B. Infective doses and pathogen carriage. Presentation at the Food Safety Education Conference, Atlanta, USDA. 2010, p:19-20. https://www.fsis.usda.gov/PDF/ Atlanta2010/S.

28. Mousavian RS, Makhdoumi KA. The inhibitory effects of static magnetic fields on Escherichia coli from two different source at short exposure time. Rep Biochem Mol Biol. 2017, 5 (2):112-116. PMID: 28367473.

29. Xu, YB, Sun SY. Effect of stable weak magnetic field on Cr (VI) bioremoval in anaerobic SBR system. Biodegradation. 2008; 19:455-462. DOI:10.1007/s10532-007-9151-5.

30. Liboff AR, Cherng S, Jenrow KA, Bull A. Calmodulin dependentcyclic nucleotide phosphodiesterase activity is altered by 20 $\mu \mathrm{T}$ magnetostatic fields. Bio Electromagn. 2003;24:32-8. https://doi.org/10.1002/bem.10063

31. Lehninger Albert L. Biochemistry- the molecular basis of cell structure and function New York: Worth Publishers Inc; 1972. ISBN 10: 0879010096

32. Dżugan M, LIS M, Droba M, Niedziółka WJ. Effect of cadmium injectted inovo on hatching results and the activity of plasma hydrolytic enzymes in newly hatched chicks. Acta Veterinaria Hungarica. 2011;59:337-347. doi.org/10.1556/AVet.2011.020.

33. Yahav S, Collin A, Shinder D, Picard M. Thermal manipulations during broiler chick embryogenesis: effects of timing and temperature. Poult Sci. 2004;83:1959-1963.

34. Attia YA, Abd El-Hamid EA, Ismaiel AM, El Nagar A, Asmaa S. The detoxification of nitrate by two antioxidants or a probiotic and the effects on blood and seminal plasma profiles and reproductive function of NZW rabbit bucks. Anim. 2013;7:591-601. doi:10.1017/S1751731112002054

35. Attia YA, Abd El-Hamid AE, El-Hanoun AM, Al-Harthi MA, AbdelRahman GM, Abdella MM. Responses of the fertility, semen quality, blood constituents, immunity and antioxidant status of rabbit bucks to type and magnetizing of water. Ann Anim Sci. 2015;15 (2):387-407. DOI: 10.2478/aoas-2015-0086

36. Al-Hassani DH, Mahbuba AG. Effect of magnetic technology of water on some productive, quality and embryonic development for broiler breeder eggs and progeny traits. (Patent). 2014, International classification A61B1/00, Iraqi classification 52. Ministry of planning/ Iraq. 